\title{
Multi-channel Phase Regenerator Based on Polarization Assisted Phase-Sensitive Amplification
}

\author{
F. Parmigiani, K. R. H. Bottrill, R. Slavík, D. J. Richardson, P. Petropoulos
}

\begin{abstract}
We propose and demonstrate simultaneous phase regeneration of six non-return-to-zero (NRZ) binary phase shift keying (BPSK) signals in a single nonlinear medium. The regenerating system which exhibits binary step-like phase transfer functions uses four-wave mixing (FWM) in degenerate dual-pump vector parametric amplifiers implemented in the same nonlinear optical fiber, followed by polarization filtering. Bit-error-ratio (BER) measurements confirm optical-signal-to-ratio (OSNR) improvement and negligible cross-talk across all the regenerated channels, which were impaired at the regenerator input by broadband phase noise.
\end{abstract}

Index Terms-Four wave mixing, nonlinear optics, phase sensitive amplifiers, multi-channel.

\section{INTRODUCTION}

Optical processing of state-of-the-art spectrally-efficient modulation format signals can potentially offer energy and cost reductions over electronic processing when advantage is taken of their ability both to process very broad optical bandwidths and many signals at the same time [1-3]. They usually also offer format flexibility and ultra-fast processing speeds. Typical examples of nonlinear optical processing functions include wavelength conversion [4], phase regeneration [5-7], electric field decomposition of phase shift keying (PSK) signals [8] and analogue-to-digital conversion (ADC) [6]. Phase sensitive amplifiers (PSAs) based on FWM effects often lie at the heart of optical subsystems developed to perform such functions. They can handle BPSK, quadrature phase shift keying (QPSK) and 16-quadrature amplitude modulation (16-QAM) signals [5-10]. However, most of the corresponding experiments so far described in the literature have accommodated a single channel and only a few attempts to simultaneously process two or more channels in a single device have been reported [9-11], highlighting the challenges faced when attempting to achieve multi-channel operation. This is often limited by the difficulty in isolating each underlying nonlinear interaction from the other for the various signals and the strong and undesired pump-to-pump (PtP) FWM interaction. This limitation may be avoided using degenerate pump configurations, in which only one pump is used and each signal must propagate over two wavelength channels (signal and signal phase conjugate idler) [9]. Indeed, by ensuring that the power of the signals is much lower than that of the pump, such a configuration has been shown capable of simultaneously amplifying up to three 16-QAM channels using a single nonlinear medium [10].

Manuscript received XXX. F. P. gratefully acknowledges the support from the Royal Academy of Engineering/EPSRC through a University research Fellowship. This research is sponsored by EPSRC grant EP/IO1196X, The Photonics Hyperhighway.

The authors are with the Optoelectronics Research Centre, University of Southampton, Southampton SO17 1BJ, U.K. (e-mail: frp@orc.soton.ac.uk).

The data for this work is accessible through the University of Southampton Institutional Research Repository (DOI:XXX).

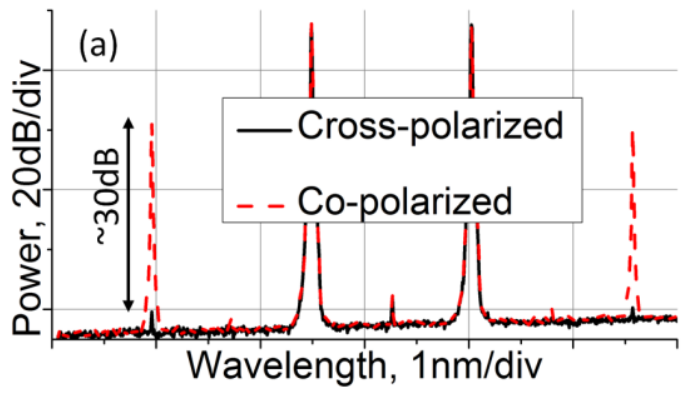

(b) PA-PSA

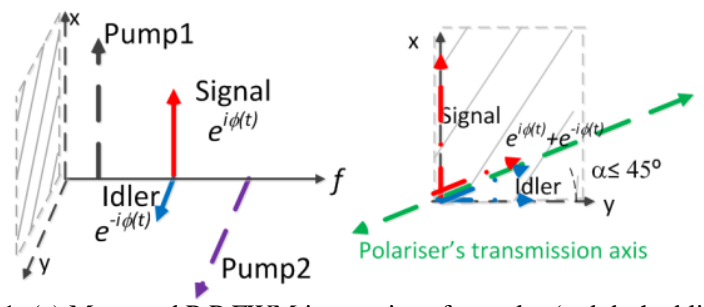

Fig.1: (a) Measured PtP FWM interactions for scalar (red dashed line) and vector (black solid line) configurations. b) Polarization assisted PSA configuration.

Although this scheme can process any modulation format, it sacrifices half of the transmission bandwidth (to transmit the idlers). Information can be carried on a single wavelength using signal degenerate dual pump configurations and regeneration of up to two channels was successfully demonstrated using two scalar PSA configurations (i.e. where the two pumps were co-polarized), sharing one pump [11]. However, in that work many undesired harmonics were generated over a broad bandwidth due to the strong pump-topump (PtP) and pump-to-signal (PtS) FWM interactions between the two PSAs. Fig.1 (a) highlights the strength of the harmonics generated by the PtP FWM interaction using a scalar configuration, see red dashed line, and how they can be reduced by as much as $30 \mathrm{~dB}$ using a vector configuration (i.e. cross-polarized pumps), see black solid line [12].

Recently we have demonstrated a Polarization-Assisted PSA (PA-PSA) scheme, based on such a vector configuration, that exhibits a high asymmetric phase sensitive extinction ratio (PSER), defined as the difference between the maximum phase sensitive (PS) -gain and the maximum PS deamplification, at modest pump powers [13]. It was used to demonstrate phase regeneration of single channel BPSK and QPSK signals [14]. Key features of the PA-PSA are that i) it uses orthogonal pumps and ii) it allows the use of low pump powers. These aspects allow minimal PtP interactions, see Fig.1 (a), and minimal PtS nonlinear interactions (which are the main cause of cross-talk under multi-channel operation) among potentially multiple PA-PSAs present in the same medium, suggesting that PA-PSAs can be spectrally packed 


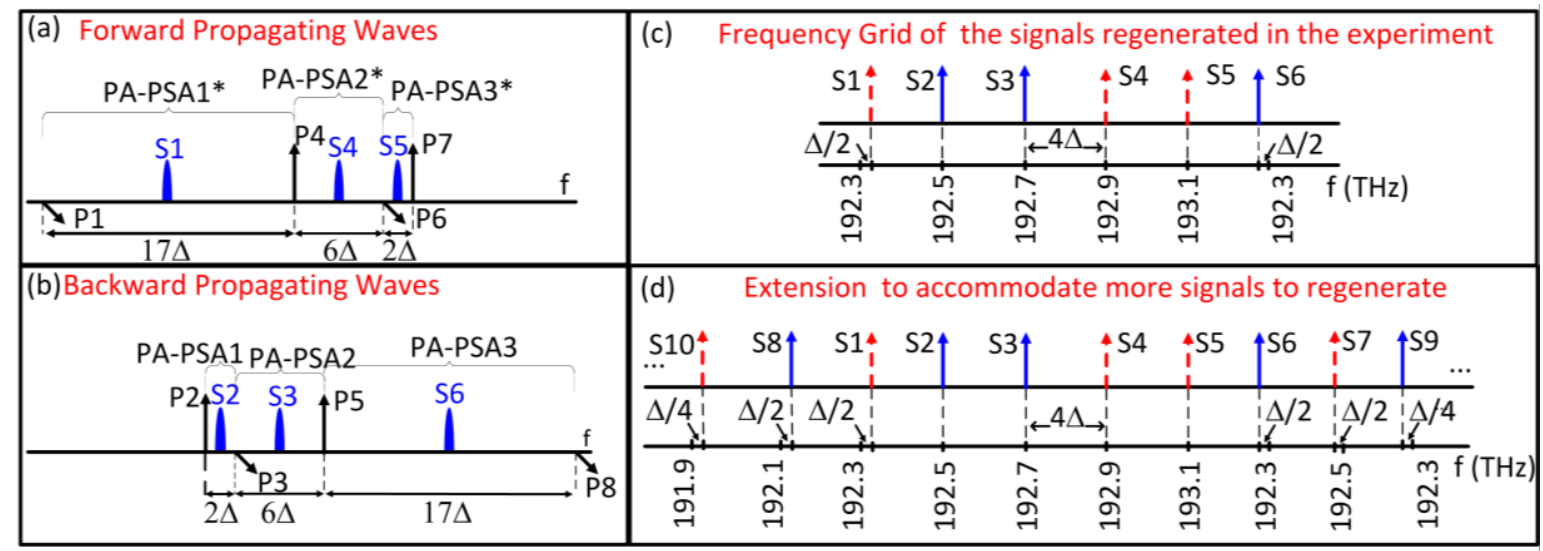

Fig.2: Pump and signal spectral locations for the forward (a) and backward (b) directions in the HNLF. $\Delta=50 \mathrm{GHz}$. Frequency grid of the regenerated signals in the actual experiment (c) and extension to a higher number of signals to regenerate (d).

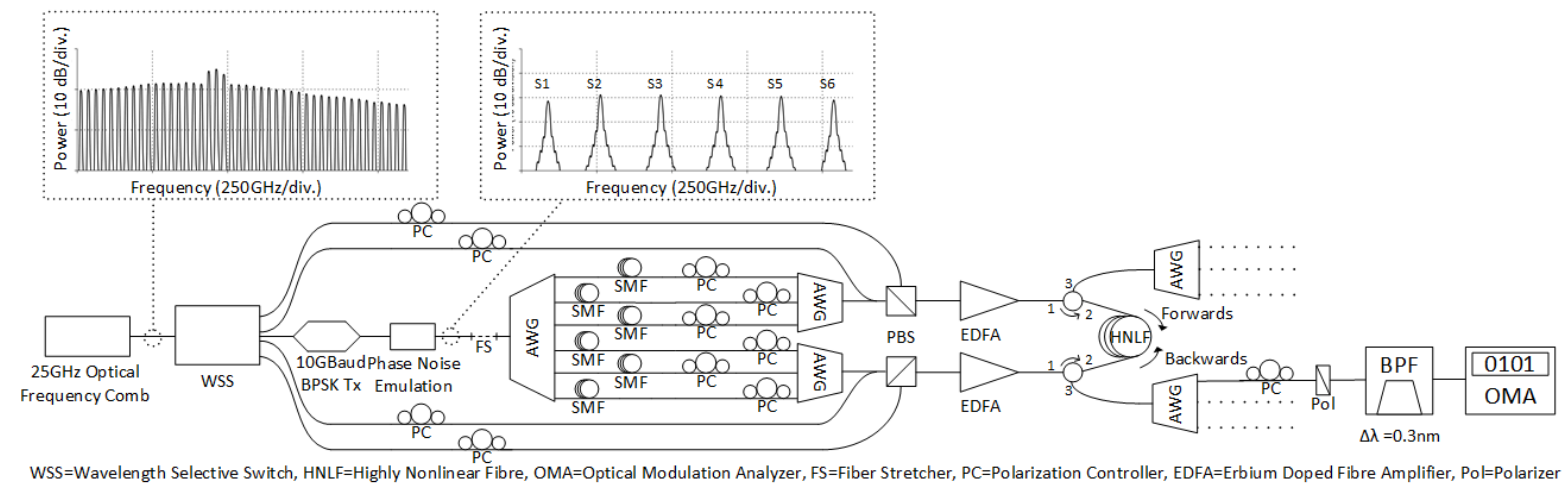

Fig.3: Experimental setup. Inset figures: spectral traces at different points of the system.

more efficiently and suffer from less cross-talk than corresponding scalar configurations. In this letter, expanding upon the results presented in [15], we benefit from these key advantages of the PA-PSA together with the use of both propagation directions in the same nonlinear medium (a highly nonlinear fiber - HNLF) as well as an engineered placement of the pump and signal wavelengths to study the simultaneous regeneration of six $10 \mathrm{Gbit} / \mathrm{s}$ BPSK wavelength division multiplexed (WDM) channels. We confirm the absense of cross-talk among the various channels and discuss extension of the scheme to a larger number of WDM channels.

\section{OPERATING PRINCIPLE}

The operation principle of the PA-PSA is illustrated in Fig.1 (b) [13-14]. The signal to be regenerated is co-polarized with one of the two orthogonally polarized phase-locked pumps, symmetrically placed in frequency about the signal. The generated idler is at the same frequency, but orthogonallypolarised to the signal, so PS operation is achieved by passing the orthogonal signal-idler pair through a polarizer with an angle, $\alpha$, selected to equalize their relative powers [13]. Thus, virtually any pump power may result in PS operation with a high PSER, provided sufficient OSNR. Fig. 2 (a) and (b) illustrates the relative spectral location of the pumps and signals in the two directions in the HNLF (effectively giving rise to three PA-PSAs along each direction) that are used in the experiment. Each PA-PSA shares one pump with its neighbouring one. The frequency spacing of the pumps in each PA-PSA needs to be different to avoid the presence of undesired scalar PSAs causing crosstalk among signals. For example, the spacing between pumps P2 and P3 (of PAPSA1) has been made three times narrower than that of P3 and P5 (of PA-PSA2). If the pumps had identical frequency spacing, then P2 and P5 would form the two pumps of a scalar PSA with S2 and S3 constituting the signal/idler pair and giving rise to severe cross-talk. As an additional measure, to avoid any overlap between the PtP and PtS FWM products among the various PA-PSAs and the signals to be regenerated, the frequency of one of them (in our specific case S1 and S6) was set to be slightly off-grid with respect to the remaining channels, as shown in Fig. 2(c). In our specific case, the frequency offset was $\Delta=25 \mathrm{GHz}$ on a nominal $200 \mathrm{GHz}$ grid. The red and blue arrows in Fig.2(c) correspond to the signals launched in the two different directions in the HNLF respectively. It is also worth noting that whereas we used phase locked signals in our experiment, making the regenerator applicable to systems using mutually coherent carriers, this is not a strict requirement of the scheme, since independent signals could also be regenerated by adopting suitable phase-locking mechanisms for the various pumps [5]. Figure 2(d) shows an example of how the scheme could potentially scale to a larger number of WDM channels. Following from above, varying offsets with respect to the exact grid frequency would have to be used for the new 
signals in order to avoid undesired overlapping between the signals to be regenerated and high order FWM harmonics. However, as the number of signals to be regenerated increases, the modulated signal bandwidth will eventually limit the scalability of the system.

\section{EXPERIMENTAL SET-UP AND RESULTS}

Figure 3 shows the experimental setup of the multi-channel phase regenerator. A $25 \mathrm{GHz}$ optical frequency comb, whose spectrum is shown in the inset to Fig. 3, guaranteed phase locking between signal and pumps of each PA-PSA without requiring the added complexity of phase-locking configurations [5]. The comb tones to constitute the pumps and signals of the various PA-PSAs were selected and demultiplexed to various paths via a wavelength selective switch (WSS), which controlled their relative power at the same time. The six lines used as the signals (at frequencies $192.325, \quad 192.5, \quad 192.7, \quad 192.9, \quad 193.1, \quad 193.275$ THz, respectively) were modulated to generate $10 \mathrm{Gbit} / \mathrm{s}$ nonreturn-to-zero (NRZ) BPSK signals, see the corresponding spectrum inset to Fig. 3. They were subsequently passed through a phase modulator (PM) for broadband phase noise emulation. The emulated noise was generated by feeding the amplified spontaneous emission noise of an erbium doped fiber amplifier (EDFA) into a photodetector. The obtained broadband electrical signal was used to drive the PM. The driving signal also contained a $70 \mathrm{kHz}$ sinusoidal dither, which was used to facilitate phase synchronization between signal and pumps in each PA-PSA. The various BPSK signals were then demultiplexed and separately propagated along optical paths of different lengths to ensure decorrelation between them, before being re-combined into two groups using two arrayed waveguide gratings (AWGs). The signals were subsequently coupled with the corresponding pumps (see Fig.2 (a) and (b)) using optical couplers and polarization beam splitters (PBSs) and launched into the two directions of the HNLF via circulators, as shown in Fig. 3. The power of each pump at the input of the HNLF was about $18 \mathrm{dBm}$, with a signal to pump ratio of $\sim-11 \mathrm{~dB}$. The strained HNLF was a

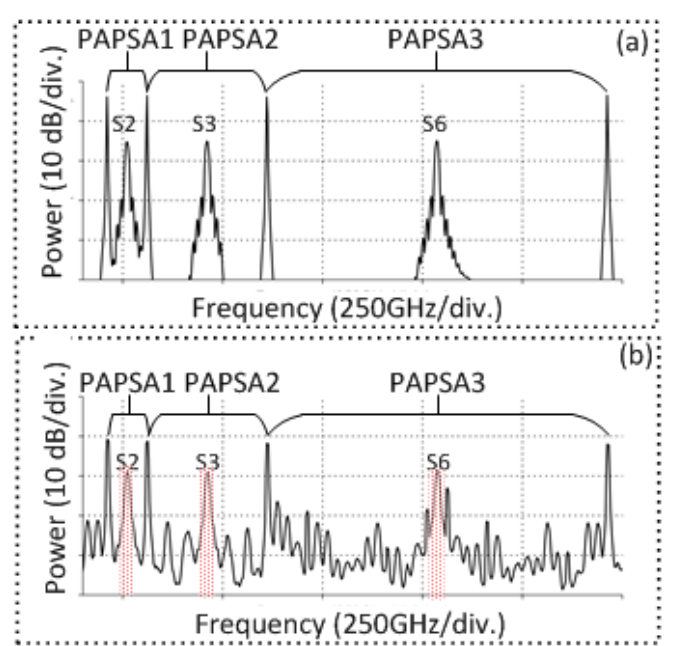

Fig.4: a) Example of spectral traces before and after the HNLF for one direction of the fiber.

302 m-long germanium-silicate fibre with a nonlinear coefficient of $11.6(\mathrm{~W} \cdot \mathrm{km})^{-1}$, a dispersion slope of 0.018 $\mathrm{ps} /\left(\mathrm{nm}^{2} \cdot \mathrm{km}\right)$, and an average zero dispersion wavelength of $1555 \mathrm{~nm}$. Typical spectra at the input and output of the HNLF are shown in Fig.4 for one direction in the fiber. Similar spectra are obtained for the other direction. In all cases, no spurious components overlap spectrally with the regenerated signals. Note that the strongest $(\sim 5 \mathrm{~dB}$ lower than the power of the S6) and closest (25 GHz far from the frequency of S6) spurious components around S6 are caused by the unmodulated (CW) PtP interactions. At the output of the HNLF, each individual channel was passed through an optical bandpass filter, a polarization controller (PC) and a polarizer, and was assessed using a pre-amplified coherent receiver and real time scope (optical modulation analyser, OMA). A small portion of each assessed output signal (not shown in the figure) was used to track thermally- and acoustically-induced phase shifts with respect to the corresponding pumps using a feedback loop that drove a fibre stretcher (FS). In the experiment, a single FS was placed in the common path of the signals. However, in practice, N FSs would be required
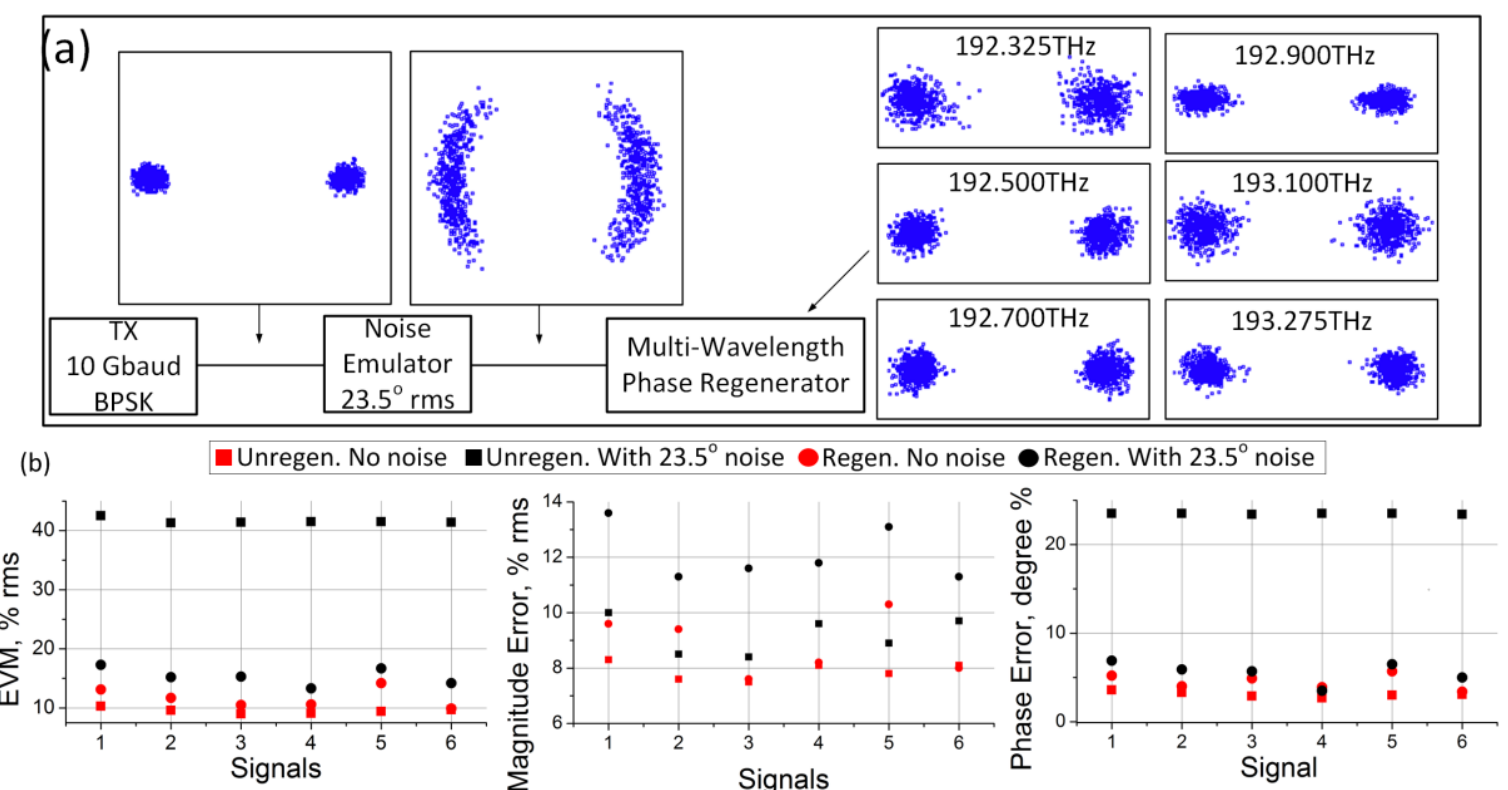

Fig.5: a) Signal constellation diagrams at different points of the system. b) Graphs of EVM (left), ME (middle), PE (right) for all the signals with and without added noise beforelafter the regenerator in multi-channel operation. 
(a)

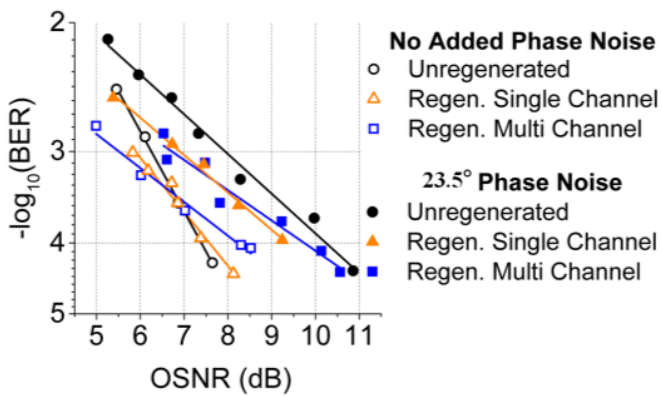

(b)

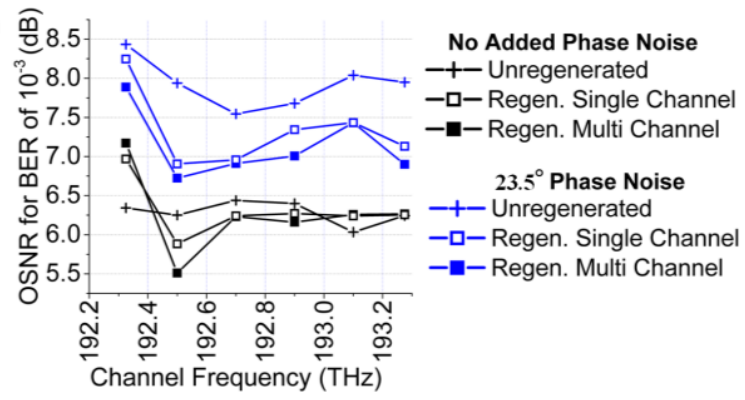

Fig.6: a) Example of BER curves withlwithout added noise beforelafter regenerator for singlelmulti-channel operation (presented for signal 192.5 THz). b) OSNRs for BER of 10-3 versus signal carrier frequency before/after the regenerator in single and multi-channel operation.

(probably located in the paths of the pumps) to simultaneously fulfil the N PSA conditions and, thus, regenerate $\mathrm{N}$ signals, increasing the overall complexity of the experimental demonstration. It is worth pointing out, though, that similar regenerative performances are expected in this scenario. The system was characterized both in single- and multi-channel operation and both without and with broadband phase noise induced. Note that in single-channel operation all the irrelevant pumps were also turned off, while ensuring the same power per pump as in the multi-channel operation. Typical examples of the constellation diagrams of the signal before and after the broadband noise emulator, and after the regenerator are shown in Fig.5 (a) under multi-channel operation. The constellation diagrams of the various signals are very similar before the regenerator and for this reason only S2 $(192.5 \mathrm{THz})$ is reported. Their corresponding error vector magnitudes (EVM), magnitude errors (ME) and phase noises (PN) are shown in Fig. 5 (b).

The full BER curves before and after the regenerator under single- and multi-channel operation, both with and without noise, are shown in Fig. 6 (a) for signal S2 as an example. Similar performance was obtained for all the channels, as shown in Fig. 6 (b), which reports the OSNR at a BER of $10^{-3}$ for all of the signals. Both in single and multi-channel operation negligible penalty was observed in the absence of noise added to the various signals. In the presence of noise, the performance of the regeneration system was very similar in single- and multi-channel operation, highlighting the absence of channel cross-talk. The maximum and minimum OSNR improvement for the multi-channel operation was 1.2 $\mathrm{dB}$ and $0.55 \mathrm{~dB}$, respectively, corresponding to signals $\mathrm{S} 2$ $(192.5 \mathrm{THz})$ and $\mathrm{S} 1$ (192.325 THz), respectively. Best regenerative performance was achieved for the central signals, most likely due to the better OSNR of the pumps in the corresponding PA-PSA at these wavelengths. This was due to the non-ideal flat-top shape of the comb source used across the utilized bandwidth (see corresponding inset in Fig.3). However, all the regenerated signals showed a performance improvement as compared to the noisy input signals.

\section{CONCLUSIONS}

We have reported the simultaneous phase regeneration of six BPSK channels using FWM-based polarization assistedPSAs. Thanks to the key features of the PA-PSA scheme (i.e. orthogonality of the pumps and low pump power), the bidirectional use of the HNLF and the choice of the relative frequency allocation of the pumps and signals, no BER penalty was observed between multi- and single-channel operation, highlighting the lack of any significant channel cross-talk. In both of the single-channel and six-channel configurations, up to $1.2 \mathrm{~dB}$ OSNR improvement for a BER of $10^{-3}$ was achieved after the regenerator when the input signals were loaded with broadband phase noise. Note that the principle can be extended to any M-PSK signals, where $\mathrm{M}$ is the number of phase levels used, in accordance to what was demonstrated in scalar configurations [6].

\section{ACKNOWLEDGMENTS}

We would like to thank OFS for supplying the HNLF.

\section{REFERENCES}

[1] P.G. Patki, et al., "Multichannel all-optical regeneration", in Photonics Society Summer Topical Meeting Series (2010), pp. 172-173.

[2] F. Parmigiani, et al., "Progress in multichannel all-optical regeneration based on fiber technology", IEEE Sel. Top. Quantum Electron. 18(2), 689-699 (2012).

[3] S. Namiki et al., "Ultrahigh-definition video transmission and extremely green optical networks for future," IEEE Journal of Selected Topics in Quantum Electronics, vol. 17, no. 2, pp. 446-457, Mar. 2011.

[4] V.J.F.Rancaño, et al., "100-GHz grid-aligned multi-channel polarization insensitive black-box wavelength converter", Journal of Lightwave Technology 2014 Vol.32 (17) pp.3027 - 3035.

[5] R.Slavík et al.,"All-optical phase and amplitude regenerator for nextgeneration telecommunications systems," Nature Photonics, vol. 4, no. 10, pp. 690-695, Oct. 2010.

[6] J. Kakande et al., "Multilevel quantization of optical phase in a novel coherent parametric mixer architecture", Nature Photonics, vol. 5, no. 12, pp. 748-752, Dec. 2011.

[7] M. Gao, et al., "Efficient phase regeneration of DPSK signal by sideband-assisted dual-pump phase sensitive amplifier", Electronics Lett., 39 (2), 140-141 (2013).

[8] M. Gao, at al., "Low-penalty Phase De-multiplexing of QPSK Signal by Dual-pump Phase Sensitive Amplifiers", ECOC, We.3.A.5 (2013).

[9] Z. Tong, et al., "Towards ultrasensitive optical links enabled by lownoise phase-sensitive amplifiers", Nature Photonics, vol. 5, pp. 430-436, 2011.

[10] T. Umeki, et al., "First demonstration of high-order QAM signal amplification in PPLN-based phase sensitive amplifier", Optics Express, vol. 22 (3), pp. 2473-2482, 2014

[11] S. Sygletos, et al., "A practical phase sensitive amplification scheme for two channel phase regeneration", Opt. Express 19, B938-B945 (2011).

[12] C. J. McKinstrie and S. Radic, "Phase-sensitive amplification in a fiber", Opt. Exp. 12, 4973-4979 (2004).

[13] F. Parmigiani, et al., "Optical phase quantizer based on phase sensitive four wave mixing at low nonlinear phase shifts", Photonics Technology Letters 2014 Vol.26(21) pp.2146-2149.

[14] F. Parmigiani, et al., "Polarization-Assisted Phase-Sensitive Processor", J. of Lightwave Technol., 33(6), 1166-1174 (2015).

[15] F. Parmigiani et al., "PSA-based all-optical multi-channel phase regenerator", accepted at ECOC 2015. 